Received: 09.06 .2018

Revised: 28.12 .2018

Accepted: 15.02 .2019

DOI: $10.17804 / 2410-9908.2019 .1 .055-063$

\title{
OPTIMAL WALL THICKNESS OF METAL PIPE ENCASED IN A FIBER POL YMER SHELL
}

\author{
S. S. Volkov ${ }^{\text {a)* }}$ and V. V. Struzhanov ${ }^{\text {b) }}$ \\ Institute of Engineering Science, Ural Branch of the Russian Academy of Sciences, \\ 34 Komsomolskaya St., 620049, Ekaterinburg, Russian Federation \\ a) (iD http://orcid.org/0000-0002-6481-5052 volkovss48@yandex.ru; \\ b) (iD http://orcid.org/0000-0002-3669-2032 国 stru@imach.uran.ru \\ *Corresponding author: E-mail: volkovss48@yandex.ru \\ Address for correspondence: 34 Komsomolskaya St., 620049, Ekaterinburg, Russian Federation \\ Tel.:+7 (343) 3623019
}

An algorithm for calculating the parameters of a compound cylinder with a metal casing and a fiber polymer shell is developed. The inner radius of the casing and the outer radius of the compound cylinder are limited by technological conditions. The compound cylinder under internal pressure deforms as a single unit. It is assumed that the thickness of the metal wall of the casing should be minimized. With the application of the Lame problem, an optimal relationship between the thickness of the casing and the thickness of its reinforcing shell is determined. Various strength conditions in the critical points of the structure are analyzed and the most comprehensive condition is chosen. An exact analytical solution of the problem is found. The ratio of two radii of a compound cylinder is found through a system of equations that relates the strength condition to the strain compatibility condition at the junction between the casing and the shell. The wall thickness for a closed cylinder with a metal casing and an open fiber polymer shell is calculated as a numerical example.

Keywords: compound cylinder, internal pressure, the Lame problem, exact solution, polymer fiber, safety factor.

\section{References}

1. Fedorov Yu.Yu., Popov S.N., Savvina A.V., Vasilyev S.V., Rodionov A.K. Evaluation of the Axial Stresses of a Gas Pipeline Made of Reinforced Polyethylene Pipes under Conditions of Permafrost Soils. Diagnostics, Resource and Mechanics of materials and structures. 2017, iss. 3, pp. 36-41. DOI: 10.17804/2410-9908.2017.3.036-041. Available at: http://dream-journal.org/issues/ 2017-3/2017-3_122. html (accessed: 22.03.2018).

2. Gorin N.V., Levakov B.G., Taskin V.B., Putyrsky V.P., Volkov S.S. Nuclear reactor vessel. RF Patent 2031457, 2002. (In Russian).

3. Struzhanov V.V., Mironov V.I. Deformatsionnoye razuprochneniye materiala v elementakh konstruktsiy [Work Softening of the Material in Structural Components]. Ekaterinburg, UrO RAN Publ., 1995, 190 p. (In Russian).

4. Andrasic C.P., Parker A.P. Dimensionless stress intensity factors for cracked thick cylinders under polynomial crack-face loading. Eng. Fract. Mech., 1984, vol. 19, no. 1, pp. 187-193.

5. Shannon R.W.E. Stress intensity factors for thick-walled cylinders. International Journal of Pressure Vessels and Piping, 1974, vol. 2, iss. 2, pp. 19-29. DOI: 10.1016/0308-0161(74)90013-1. 
6. Lavit I.M., Nguyen V.T. Thermoelastoplastic deformation of a thick-walled cylinder with a radial crack. Journal of Applied Mechanics and Technical Physics, 2008, vol. 49, iss. 3, pp 491-499. DOI: $10.1007 / \mathrm{s} 10808-008-0066-7$.

7. Zingerman K. M., Zubov L. M. Exact solutions of problems of the theory of repeated superposition of large strains for bodies created by successive junction of strained parts. Chebyshevskii Sb., 2017, vol. 18, iss. 3, pp. 255-279. (In Russian).

8. Timoshenko S.P., Goodier J.N. Theory of Elasticity, New York, Toronto, London, McGrawHill Book Company, 1951.

9. Timoshenko S.P., Gere J.M. Mechanics of Materials, New York, Von Norstrand, Reinhold Co., 1972.

10. Birger I.A., Mavlyutov R.R. Soprotivlenie materialov [Strength of Materials]. Moscow, Nauka Publ., 1986, 560 pp. (In Russian). 
Подана в журнал: 09.06.2018

УДК 539.3

DOI: $10.17804 / 2410-9908.2019 .1 .055-063$

\title{
ОПТИМАЛЬНАЯ ТОЛЩИНА СТЕНКИ МЕТАЛЛИЧЕСКОЙ ТРУБЫ С ВОЛОКНИСТОЙ ОБОЛОЧКОЙ
}

\author{
С. С. Волков ${ }^{\text {a)* }}$, В. В. Стружанов ${ }^{\text {б) }}$ \\ Федеральное государственное бюджетное учреждение науки Институт машиноведения \\ Уральского отделения Российской академии наук \\ 620049, ул. Комсомольская, 34, Екатеринбург, Российская Федерация \\ a) (D) http://orcid.org/0000-0002-6481-5052 olkovss48@yandex.ru; \\ б) (iD http://orcid.org/0000-0002-3669-2032 ख stru@imach.uran.ru \\ *Ответственный автор. Электронная почта: volkovss48@ yandex.ru \\ Адрес для переписки: ул. Комсомольская 34, Екатеринбург, Российская Федерация \\ Тел.: +7 (343) 362-30-19
}

\begin{abstract}
Разработан алгоритм расчета параметров составного цилиндра из металлического корпуса и полимерной волокнистой оболочки. Внутренний радиус корпуса и внешний радиус составного цилиндра ограничены технологическими условиями. Составной цилиндр деформируется как единое целое под действием внутреннего давления. Предполагается, что толщина металлической стенки корпуса должна быть наименьшей из возможных. С помощью решения задачи Ламе находится оптимальное соотношение между толщиной стенки корпуса и толщиной упрочняющей его оболочки. Проведен анализ различных условий прочности в опасных точках конструкции и выбрано наиболее общее из условий. Найдено точное аналитическое решение задачи. Соотношение радиусов составного цилиндра находится при решении системы уравнений, связывающих условие прочности и условие совместности деформаций на стыке корпуса и оболочки. Приведен числовой пример расчета толщины стенок для закрытого цилиндра с металлическим корпусом и открытой волокнистой оболочкой.
\end{abstract}

Ключевые слова: составной цилиндр, внутреннее давление, задача Ламе, точное решение, полимерное волокно, запас прочности.

\section{1. Введение}

Цилиндрические элементы конструкций, в том числе составные цилиндры, активно используются в технике. Это резервуары различного назначения, трубопроводы, котлы, корпусы двигателей. Разработка методов расчета составных цилиндров на прочность становится актуальной и с появлением новых современных материалов $[1,2]$. При этом ставятся задачи прогнозирования ранних стадий разрушения материала и гарантирование работы ответственных конструкций в безопасном диапазоне напряжений и деформаций [3-6]. Наряду с созданием пакетов программ для расчета конструкций сохраняется необходимость и в разработке точного аналитического решения задач [7].

\section{2. Постановка задачи}

Рассмотрим цилиндр, боковая поверхность которого имеет полимерную волокнистую оболочку. Цилиндр находится под воздействием высокого внутреннего давления, имеющего характер однократных статических нагрузок. Требуется оценить прочность боковой поверхности. Необходимо найти наименьшую из возможных толщину корпуса, не допустив при этом потери прочности конструкции. Суммарная толщина корпуса и оболочки ограничена технологическими требованиями. 
Внутренний радиус корпуса $a$ известен. Его внешний радиус $b$ и внешний радиус оболочки $c$ не известны и должны быть найдены так, чтобы при действии давления внутри цилиндра не возникали пластические деформации ни в корпусе, ни в оболочке. При возрастании давления внутри цилиндра до величины $P_{a}$ на стыке корпуса и оболочки возникает давление $P_{b}$. Величина $P_{b}$ зависит от соотношения радиусов $a, b, c$, поэтому заранее неизвестна.

Оболочка плотно прилегает к корпусу, напряжения на стыке корпуса и оболочки при отсутствии внутреннего давления практически отсутствуют. Подобные оболочки надеваются на корпус с натягом, но релаксация высокопрочных волокон обычно такова, что эти напряжения быстро снижаются, поэтому их можно не учитывать при расчете ответственных конструкций.

На рис. 1 представлена схема поперечного сечения составного цилиндра. Толщина металлической стенки корпуса равна $b-a$, толщина полимерной обмотки равна $c-b$.

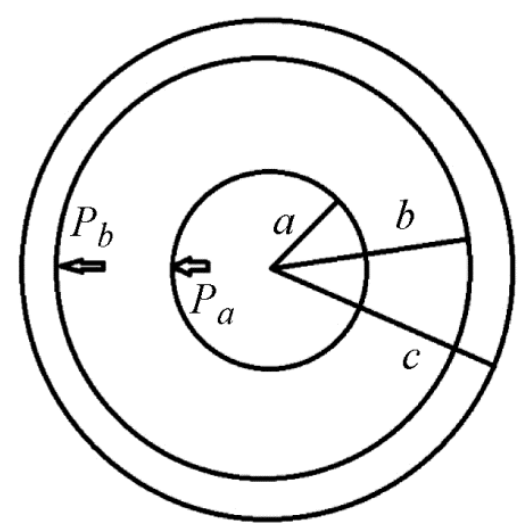

Рис. 1. Схема сечения составного цилиндра

Пусть $E_{1}-$ модуль Юнга, $v$ - коэффициент Пуассона материала корпуса. $E_{2}-$ модуль Юнга волокна полимерной оболочки. Обмотка не имеет связующего, поэтому ее коэффициент Пуассона равен нулю.

Рассмотрим решение задачи Ламе для металлического корпуса и оболочки из волокна. Корпус - цилиндр с внутренним давлением $P_{a}$ и внешним давлением $P_{b}$ на боковой поверхности. Оболочка представляет собой цилиндр с внутренним давлением $P_{b}$. Внешнее давление оболочки $P_{c}$ равно нулю.

\section{3. Решение задачи Ламе}

В корпусе действуют $\sigma_{r}$ - радиальное, $\sigma_{\theta}$ - окружное и $\sigma_{z}-$ осевое напряжения. Соответствующие напряжения в оболочке обозначим $\tilde{\sigma}_{\theta}, \tilde{\sigma}_{r}, \tilde{\sigma}_{z}$. Пусть также $r$ - произвольный радиус сечения составного цилиндра, расстояние от оси цилиндра до его произвольной точки.

Используя методы решения [8-10] задачи Ламе для цилиндра, запишем напряжения в металлическом корпусе при $a \leq r \leq b$ :

$$
\left.\begin{array}{c}
\sigma_{\theta}=\frac{a^{2} P_{a}-b^{2} P_{b}}{b^{2}-a^{2}}+\frac{P_{a}-P_{b}}{b^{2}-a^{2}} \frac{a^{2} b^{2}}{2}, \\
\sigma_{r}=\frac{a^{2} P_{a}-b^{2} P_{b}}{b^{2}-a^{2}}-\frac{P_{a}-P_{b}}{b^{2}-a^{2}} \frac{a^{2} b^{2}}{2}, \\
\sigma_{z}=\frac{a^{2} P_{a}}{b^{2}-a^{2}} .
\end{array}\right\} .
$$


Аналогичным образом найдем радиальное, окружное и осевое напряжения в волокнистой оболочке при $b \leq r \leq c$ :

$$
\left.\begin{array}{c}
\tilde{\sigma}_{\theta}=\frac{b^{2} P_{b}}{c^{2}-b^{2}}+\frac{P_{b}}{c^{2}-b^{2}} \cdot \frac{b^{2} c^{2}}{r^{2}}, \\
\tilde{\sigma}_{r}=\frac{b^{2} P_{b}}{c^{2}-b^{2}}-\frac{P_{b}}{c^{2}-b^{2}} \cdot \frac{b^{2} c^{2}}{r^{2}}, \\
\tilde{\sigma}_{z}=0 .
\end{array}\right\} .
$$

Используя обобщенный закон Гука, вычислим окружные деформации в корпусе $\varepsilon_{\theta}$ и оболочке $\widetilde{\varepsilon}_{\theta}$ при $r=b$ :

$$
\begin{aligned}
& E_{1} \cdot \varepsilon_{\theta}=\sigma_{\theta}-v\left(\sigma_{r}+\sigma_{z}\right)=\frac{P_{a}(2-v) a^{2}-P_{b}(1-v) b^{2}-P_{b}(1+v) a^{2}}{b^{2}-a^{2}}, \\
& E_{2} \cdot \tilde{\varepsilon}_{\theta}=\tilde{\sigma}_{\theta}=P_{b} \frac{c^{2}+b^{2}}{c^{2}-b^{2}} .
\end{aligned}
$$

Запишем условие совместности деформаций $\varepsilon_{\theta}=\widetilde{\varepsilon}_{\theta}$ при $r=b$ на стыке корпуса и оболочки:

$$
\frac{P_{a}(2-v) a^{2}-P_{b}(1-v) b^{2}-P_{b}(1+v) a^{2}}{E_{1}\left(b^{2}-a^{2}\right)}=P_{b} \frac{c^{2}+b^{2}}{E_{2}\left(c^{2}-b^{2}\right)}
$$

Введем обозначения:

$$
x=\frac{b^{2}}{a^{2}}, \quad y=\frac{c^{2}}{b^{2}}, \quad z=\frac{P_{b}}{P_{a}} .
$$

Используя обозначения (3), получим условие совместности деформаций на стыке корпуса и обмотки в следующем виде:

$$
\frac{2-v-z(1-v) x-z(1+v)}{E_{1}(x-1)}=\frac{z}{E_{2}} \cdot \frac{y+1}{y-1} .
$$

Условие совместности деформаций должно сочетаться с ограничением напряжений в опасных точках и отсутствием в них пластических деформаций.

\section{4. Условия прочности корпуса и оболочки}

Перейдем к оценке напряжений в составном цилиндре, используя формулы $(1,2)$. Наибольшие напряжения, влияющие на прочность конструкции, возникают на внутренней поверхности корпуса при $r=a$ и на внутренней поверхности оболочки при $r=b$. Для выбора критерия прочности рассмотрим также интенсивность напряжений $\sigma_{i}$ и главные касательные напряжения $\tau_{1}, \tau_{2}, \tau_{3}$ в опасных точках корпуса и оболочки: 


$$
\sigma_{i}=\sqrt{\frac{1}{2}\left(\left(\sigma_{\theta}-\sigma_{r}\right)^{2}+\left(\sigma_{r}-\sigma_{z}\right)^{2}+\left(\sigma_{z}-\sigma_{\theta}\right)^{2}\right)} .
$$

Касательные напряжения $\tau_{1}, \tau_{2}, \tau_{3}$ в корпусе при $r=a$ :

$$
\begin{aligned}
& \tau_{1}=\sigma_{\theta}-\sigma_{r}=2 P_{a}(1-z) \cdot \frac{x+1}{x-1}, \\
& \tau_{2}=\sigma_{z}-\sigma_{r}=P_{a} \cdot \frac{x}{x-1}, \\
& \tau_{3}=\sigma_{\theta}-\sigma_{z}=P_{a} \cdot \frac{x(1-2 z)}{x-1} .
\end{aligned}
$$

Вычислим интенсивность касательных напряжений в корпусе при $r=a$ :

$$
\sigma_{i}=\frac{P_{a}}{x-1} \sqrt{2(1-z)^{2}(x+1)^{2}+x^{2}\left(1-2 z+2 z^{2}\right)} .
$$

Анализируя напряжения $\sigma_{i}, \tau_{1}, \tau_{2}, \tau_{3}$, делаем вывод, что в корпусе наибольшим из них является $\tau_{1}$. Если $\left[\sigma_{i}\right]$ - допустимое значение интенсивности напряжений в металле, то условие прочности корпуса при минимальной толщине металлической стенки примет вид:

$$
2(1-z) \cdot \frac{x+1}{x-1}=\frac{\left[\sigma_{i}\right]}{P_{a}}
$$

Аналогичным образом исследуем напряжения $\tilde{\tau}_{1}, \tilde{\tau}_{2}, \tilde{\tau}_{3}$ для оболочки при $r=b:$

$$
\begin{aligned}
& \tilde{\tau}_{1}=\tilde{\sigma}_{\theta}-\tilde{\sigma}_{r}=2 z P_{a} \cdot \frac{y+1}{y-1}, \\
& \tilde{\tau}_{2}=\tilde{\sigma}_{z}-\tilde{\sigma}_{r}=z P_{a}, \\
& \tilde{\tau}_{3}=\tilde{\sigma}_{\theta}-\tilde{\sigma}_{z}=z P_{a} \cdot \frac{\sqrt{2,5 y^{2}+y+1}}{y-1} .
\end{aligned}
$$

Если $\left[\tilde{\sigma}_{i}\right]$ - допустимое значение интенсивности напряжений волокна, то условие работы оболочки в области упругих деформаций следующее:

$$
2 z \cdot \frac{y+1}{y-1}=\frac{\left[\tilde{\sigma}_{i}\right]}{P_{a}} .
$$

И наконец, учитывая суммарное действие корпуса и оболочки, запишем напряжения в опасных внутренних точках корпуса при $r=a$ : 


$$
\sigma_{\theta}=P_{a} \frac{x y+1}{x y-1}, \quad \sigma_{r}=-P_{a}, \quad \sigma_{z}=P_{a} \frac{1}{x-1} .
$$

Для условия прочности используем максимальное касательное напряжение

$$
\sigma_{\theta}-\sigma_{r}=P_{a} \cdot \frac{2 x y+1}{x y-1} \leq\left[\sigma_{i}\right]
$$

или интенсивность напряжений:

$$
\sigma_{i}=P_{a} \cdot \frac{x}{(x y-1)(x-1)} \sqrt{y^{2}\left(3 x^{2}-6 x+4\right)+1-2 y} \leq\left[\sigma_{i}\right] .
$$

Для проведения дальнейших расчетов исключим параметр $z$ в формуле (4), используя выражение (5). В результате получим соотношение между неизвестными $x$ и $y$.

$$
2 \frac{(x-1)(S-v)-v}{(x-1)(S-x S-2 x)}=v+\frac{E_{1}}{E_{2}}\left(1+\frac{2}{y-1}\right) ; S=\frac{\left[\sigma_{i}\right]}{P_{a}} .
$$

\section{5. Уравнения для расчета оптимального соотношения радиусов цилиндра}

Результаты расчетов показывают, что для заданного диапазона изменения $x$ и $y$ критерий (7) сильнее, чем критерий (6). Анализ показывает также, что условие (8) является более общим, чем условие (4). Таким образом, решение задачи об оптимальной толщине корпуса и обмотки должно осуществляться по формулам (8) и (9).

Запишем искомую систему двух уравнений для определения неизвестных $x$ и $y$ :

$$
\left\{\begin{array}{c}
P_{a} \cdot \frac{x}{(x y-1)(x-1)} \sqrt{y^{2}\left(3 x^{2}-6 x+4\right)+1} \leq\left[\sigma_{i}\right] \\
2 \frac{(x-1)\left(\left[\sigma_{i}\right]-v P_{a}\right)-v P_{a}}{(x-1)\left(\left[\sigma_{i}\right]-x\left[\sigma_{i}\right]-2 x P_{a}\right)}=v+\frac{E_{1}}{E_{2}}\left(1+\frac{2}{y-1}\right)
\end{array} .\right.
$$

Решив систему уравнений (10), вычислим неизвестные внешний радиус металлического корпуса $b$ и внешний радиус полимерной оболочки $c$. Радиус $a$ известен из условий задачи

$$
b=a \sqrt{x} ; \quad c=b \sqrt{y}=a \sqrt{x y} .
$$

Находим также толщину стенки корпуса $b-a$ и толщину оболочки $c-b$ :

$$
b-a=a(\sqrt{x}-1) ; c-b=b(\sqrt{y}-1) .
$$




\section{6. Числовой пример}

Цилиндрический корпус изготовлен из титанового сплава ВТ-6 и упрочнен обмоткой из волокна армос. Модуль Юнга сплава $E_{1}=1,04 \cdot 10^{5} \mathrm{MПа;} \mathrm{модуль} \mathrm{Юнга} \mathrm{волокон} E_{2}=$ $=1,26 \cdot 10^{5}$ МПа. Коэффициент Пуассона для корпуса $v=0,33$, для оболочки $-v=0$, так как обмотка не имеет связующего. В результате кратковременного воздействия в металлическом корпусе возникает внутреннее давление $P_{a}=100$ МПа. Внутренний радиус корпуса $a=190$ мм. Согласно технологическим условиям $c \leq 1,41 a$, т. е. неизвестные параметры $x$ и $y$ связаны условием $x \cdot y \leq 1,89$. Нужно найти размеры корпуса и оболочки так, чтобы работа конструкции была безопасной, а толщина металлической стенки - наименьшей из возможных.

Допустимое напряжение $\left[\sigma_{i}\right]$ в металле свяжем с пределом текучести титанового сплава $\sigma_{T}=880$ МПа и различными значениями коэффициента запаса прочности $[n]$, тогда $\left[\sigma_{i}\right]=$ $\sigma_{T} /[n]$. Допустимое напряжение волокна $\left[\tilde{\sigma}_{i}\right]=4400$ МПа. Условия прочности достаточно рассмотреть лишь для металла, так как прочность волокна в несколько раз больше. мут вид:

Если $[n]-$ коэффициент запаса прочности, то условия для расчета величин $x$ и $y$ при-

$$
\left\{\begin{array}{c}
\frac{x}{(x y-1)(x-1)} \sqrt{y^{2}\left(3 x^{2}-6 x+4\right)+1} \leq \frac{8,8}{[n]}, \\
2 \frac{(x-1)(8,8-v[n])-v[n]}{(x-1)(8,8-x 10,8)}=v+0,82\left(1+\frac{2}{y-1}\right) .
\end{array}\right.
$$

На рис. 2 представлено графическое решение системы (12). Решение $A(1,23 ; 1,40)$ получено при коэффициенте запаса прочности $[n]=2$. При меньшем запасе прочности $[n]=1,5$ получается решение $B(1,19 ; 1,29)$.

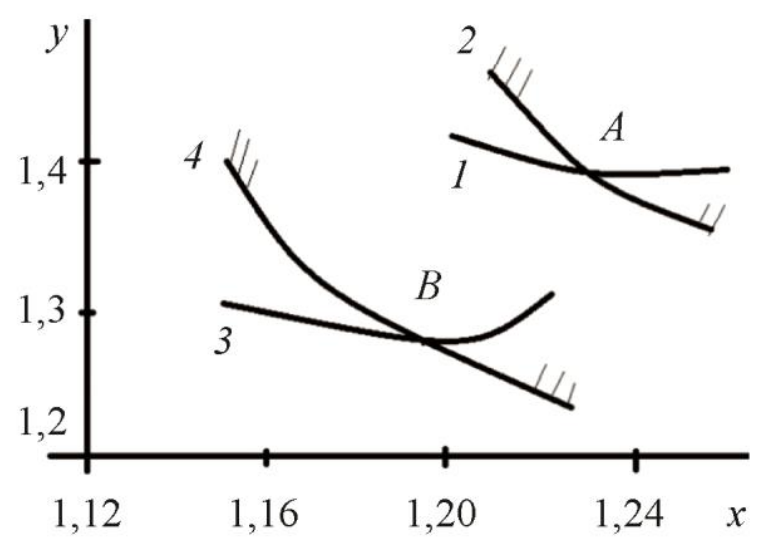

Рис. 2. Решение $(x, y)$ при различных коэффициентах запаса прочности: $A-[n]=2$; $B-[n]=1,5$ (линии $1,3-$ условия совместности деформаций; линии 2, 4 - условия прочности)

Рассмотрим точку $A$. Здесь $x=1,23$ и $y=1,40$. По формулам (11) толщина стенки корпуса равна $a(\sqrt{x}-1)=21$ мм, толщина оболочки: $b(\sqrt{y}-1)=38$ мм. Решение в точке $B$ дает, соответственно, более тонкие корпус и оболочку: 17 мм и 29 мм. Корпус и оболочка действуют при этом в безопасной области упругих напряжений и деформаций. 


\section{7. Заключение}

Получено точное аналитическое решение для расчета толщины стенок составного цилиндра из металлического корпуса и полимерной волокнистой оболочки в безопасной области упругости. Используются различные коэффициенты запаса прочности. При необходимости учесть динамический характер внутреннего давления расчеты могут проводиться с соответствующим коэффициентом, завышающим запас прочности. Полученные формулы дают возможность делать расчеты в широком диапазоне деформационных и прочностных свойств материала корпуса и материала оболочки, а также исходных размеров цилиндра. Результаты могут быть использованы для тестирования программ численного моделирования цилиндрических сосудов с оболочкой.

\section{Литература}

1. Evaluation of the Axial Stresses of a Gas Pipeline Made of Reinforced Polyethylene Pipes under Conditions of Permafrost Soils / Yu. Yu. Fedorov, S. N. Popov, A. V. Savvina, S. V. Vasilyev, A. K. Rodionov // Diagnostics, Resource and Mechanics of materials and structures. - 2017. - Iss. 3. P. 36-41. - DOI: 10.17804/2410-9908.2017.3.036-041. - URL: http://dream-journal.org/issues/ 2017-3/2017-3_122.html (accessed: 22.03.2018).

2. Корпус ядерного реактора : пат. 2031457 Рос. Федерация / Горин Н. В., Леваков Б. Г., Таскин В. Б., Путырский В. П., Волков С. С., РФЯЦ-ВНИИТФ НПП «Уралкомплекс», Снежинск. - № 4934045/25 ; заявл. 06.05.1991 ; опубл. 20.03.95, Бюл. № 8. -5 с.

3. Стружанов В. В. Миронов В. И. Деформационное разупрочнение материала в элементах конструкций. - Екатеринбург : УрО РАН, 1995. - 190 с.

4. Andrasic C. P., Parker A. P. Dimensionless stress intensity factors for cracked thick cylinders under polynomial crack-face loading // Eng. Fract. Mech. - 1984. - Vol. 19, no. 1. - P. 187-193.

5. Shannon R. W. E. Stress intensity factors for thick-walled cylinders // International Journal of Pressure Vessels and Piping. - 1974. - Vol. 2, iss. 2. - P. 19-29. - DOI: 10.1016/03080161(74)90013-1.

6. Lavit I. M., Nguyen V. T. Thermoelastoplastic deformation of a thick-walled cylinder with a radial crack // Journal of Applied Mechanics and Technical Physics. - 2008. - Vol. 49, iss. 3. P. 491-499. - DOI: 10.1007/s10808-008-0066-7.

7. Зингерман К. М., Зубов Л. М., Точные решения задач теории многократного наложения больших деформаций для тел, образованных последовательным соединением деформированных частей // Чебышевский сборник. - 2017. - Т. 18, вып. 3. - С. 255-279.

8. Timoshenko S. P., Goodier J. N. Theory of Elasticity. - New York, Toronto, London : McGraw-Hill Book Company, 1951.

9. Timoshenko S. P., Gere J. M. Mechanics of Materials. - New York : Von Norstrand, Reinhold Co., 1972.

10. Биргер И. А., Мавлютов Р. Р. Сопротивление материалов. - М. : Наука, 1986. - 560 с. 